\title{
La controversia entre homosexualidad y familia: el caso cubano
}

\section{Luis Robledo Díaz}

Universidad de La Habana

robledodiaz@yahoo.es

\section{Resumen}

El presente artículo constituye un resumen de la tesis Homosexualidad y familia: acoso y simetrías que el autor presentara para obtener el título de máster en Sociología por la Universidad Autónoma de Barcelona y la Universidad de La Habana. En la misma nos propusimos caracterizar algunos aspectos de la relación entre homosexualidad y familia en el contexto cubano. Para ello, nos centramos en describir los aspectos relativos a la "vida gay» en Ciudad de La Habana y en determinar el vínculo establecido entre homosexualidad y familia por un grupo de jóvenes homosexuales varones residentes en esta ciudad.

Palabras clave: sexualidad, homosexualidad, familia, género, Cuba, sociología.

Abstract. Controversy homosexuality and family: the cuban case

The present article constitutes a summary of the thesis Homosexuality and family: harassment and symmetries that author presented to obtain the title of Masters in Sociology by the Independent University of Barcelona and the University of Havana. We seted out to characterize some aspects of the relation between homosexuality and family in the Cuban context. For it we were centred in describing to the aspects relative to the «life gay» in Havana City and determining the bond established between homosexuality and family by a group of homosexual young man people resident in this city.

Key words: sexuality, homosexuality, family, gender, Cuba, sociology.

\section{Sumario}

Introducción Conclusiones

Elementos para el debate Bibliografía

El caso cubano 


\section{Introducción}

La arquitectura falocrática dominante sigue empecinada en acostumbrarnos a observar su maqueta como la cumbre de la civilización humana y para ello acosa cualquier acto que pudiera constituir una vejación a este orden. La homosexualidad y los homosexuales se han visto envueltos, a lo largo del tiempo, en esta maraña. Con un origen perdido - ya sea en la historia o en la ciencia-, a esta forma de expresión de la sexualidad y a aquéllos que la experimentan se les ha hundido en un mar de intrigas y maquinaciones, y se les acosa para obligarlos a simular la efectividad de los tratamientos paliativos a su «desorden conductual». Como singular acólito: la hoguera, la cárcel y las terapias médicas.

Pero tal efectividad parece, cada vez más, entrar en el campo de la duda. Sobre ello reflexionamos en la tesis Homosexualidad y familia: acoso y simetrias ${ }^{1}$, donde concebimos un modelo de argumentación sobre la pertinencia del estudio de la homosexualidad como objeto sociológico y tratamos de desestructurar la noción popular y con cierto predominio también en el campo de las ciencias sociales cubanas - y en los propios científicos- de los enlaces unilineales tradicionalmente hechos entre sexo, género y orientación sexual. No nos propusimos «estructurar» un nuevo enlace, sino demostrar la tantas veces dicha pero pocas veces tratada y asumida concepción pluridimensional de la construcción del yo sexual en la especie humana. Era menester, antes que cualquier otra propuesta, traer a debate un tema con una verdad aún oculta en Cuba.

Al ser una zona prácticamente inexplorada dentro del universo científico la Isla, la variedad de análisis era —es, todavía — muy amplia, amén de atrasada con respecto al desarrollo de los estudios en otros países. Al avanzar en la encrucijada del debate sobre la homosexualidad actualmente en el mundo, nos dimos cuenta de que uno de los puntos más álgidos era la relación de la homosexualidad con el orden familiar hegemónico. Nos pareció así interesante y un buen objeto de estudio explorar en torno al comportamiento de esta temática en nuestro país. Sobre la controversia entre homosexualidad y familia y la forma en que se han enlazado estas dos categorías, dedicamos la segunda parte de dicha investigación. Ello nos sirvió de base para estructurar tanto el diseño como el posterior análisis de los resultados obtenidos en el trabajo de campo. Éste último se concibió en dos momentos: 1) descripción de la «vida gay» de jóvenes homosexuales varones en Ciudad de La Habana; y 2) la relación entre homosexualidad y familia desde los propios homosexuales varones.

\section{Elementos para el debate ${ }^{2}$}

Juan Antonio Blanco (1995) observa dentro de los asertos principales de la visión de la modernidad el de considerar a «la familia basada en el esquema

1. Véase Robledo, 2000.

2. En la presente nota de investigación, reseñaremos los dos últimos capítulos, dado que en éstos se concentran las bases que cumplimentan los objetivos centrales del estudio. 
patriarcal, monógamo y heterosexual [como] el modo superior y final de organización antropológica» (1995: 26). La familia ha corrido así una suerte similar a otras categorías como las de sexo y género: el de ser considerada como ahistórica, eterna e inmutable.

Pero la realidad viene demostrando dos cosas: a) la inexistencia de un modelo único y universal de familia; y $b$ ) la emergencia de valores que vienen a poner en crisis dicho modelo hegemónico. La familia — valga decirlo- no existe, sino como historicidad, como construcción cultural.

Quizás en algunos sectores más que en otros esta concepción de amplitud encuentre espacios más favorables de comprensión. Hoy se acepta la noción no sólo de familia matrimonial, sino también consensual, monoparental, ensamblada o reconstituida; la existencia en distintos períodos históricos e incluso coincidir temporalmente de sociedades patriliniales o matriliniales, organizaciones poligámicas (poliándricas y poligínicas), etc. Se habla de familias invisibles por la no existencia aún de una nominación particular adecuada a determinados tipos emergentes en la actualidad. Se discute la pertinencia de utilizar determinados criterios como base para las tipificaciones históricas de los distintos modelos de familia y la convivencia de éstos en la contemporaneidad, no sólo como tipos puros, sino presentes en una misma familia. Pero la heterosexualidad no se cuestiona. Sólo recientemente, tras la lucha de distintos grupos de gays y lesbianas, ha entrado en debate el tema de la constitución de familias cuyos miembros sean homosexuales (por su sexo y por sus relaciones sexoeróticas si pensamos en pareja) y la posibilidad de tenencia y cuidado de una descendencia.

Sin embargo, la controversia entre homosexualidad y familia no empieza en este punto. Existen varias teorías que tras la certeza de una causa adquirida, veían en la familia la génesis de tal conducta o condición. Es éste uno de los enlaces; el otro, es la noción de homosexualidad como destructora del orden familiar y peligro de extinción de la especie - y la cultura - por ser hecho contra natura y no procrear. Un tercer enlace es el que convierte a la familia en impedimento para el desarrollo de una identidad coherente con la orientación sexual del adolescente o joven si éste es homosexual.

1. Varias teorías han concebido el origen de la homosexualidad como una malformación de la función educativa de la familia

Alfred Adler (1930) reduce el problema de la homosexualidad a la incapacidad de resolver el miedo hacia el otro sexo. En el miedo al fracaso, Adler ve «la estructuración de la perversión como seguridad, que lleva a cabo con la finalidad de fijar de una vez para siempre la distancia que separa al individuo de su compañero (o compañera) sexual» (1930: 3). Todo ello tiene su explicación última en esquemas establecidos durante la infancia. Según esta teoría, en la misma medida que el niño va creciendo, se van desarrollando en él «tendencias anímicas hacia el poderío y el hacerse valer». Para ello se elige un «ideal director» frecuentemente representado por la madre o el padre. Esta figura sirve de referente para medir sus propias capacidades desde el cual articula, además, estra- 
tegias de resolución de conflictos en su vida futura. Sin embargo, existe el riesgo de ocurrencia de «errores» educativos que lleven a un sentimiento de inferioridad en el niño. Ello pudiera estar producido por la presencia exagerada de una dominación paterna o la existencia de un padre débil frente a una madre dominante. En cualquier caso, ello hace infranqueable al niño su distancia frente a los adultos. Este sería el origen de la incongruencia entre su conducta (anormal) y las normas de la sociedad (1930: 39).

La falta de consistencia teórica de estos postulados radica en la sobredeterminación de roles paterno y materno, o de lo femenino y lo masculino, como si fueran dados por lo natural, y en una sobredeterminación de lo simbólicamente masculino dentro del "proyecto de vida». ${ }^{3}$ Wilhelm Stekel (1924) critica este exceso de importancia que le brinda Adler al contraste entre lo femenino y lo masculino. Stekel, seguidor del psicoanálisis de Freud, sostiene el criterio de la inexistencia de una homosexualidad o heterosexualidad en estado puro y, por tanto, la imposibilidad de conducir el estudio hacia la búsqueda de un origen congénito de tal condición. La monosexualidad no existe, o al menos no es normal; lo normal es la disposición bisexual y ésta es congénita. Bajo determinadas circunstancias, y por motivos múltiples, algunos individuos reprimen uno de sus dos componentes (el heterosexual o el homosexual) y aparecen entonces como monosexuales. Dada la multiplicidad de causales y caminos posibles para llegar al origen de la homosexualidad, Stekel ve poco apropiado reducir todo este proceso a las influencias de orden familiar, aunque no niega la importancia que éstas revisten en la explicación de dicha condición. Así, dicho origen puede ser encontrado desde la existencia de «trastornos» similares en algún otro miembro de la familia hasta, por ejemplo, el hecho de que el padre sea mujeriego, en tanto podría producirse un deseo de diferenciación del hijo con relación a su progenitor (1920: 364). También acepta las distintas variantes en el proceso de identificación, sea éste con un ideal masculino (padre, hermano, etc.) o femenino (madre o prostituta). Ello podría, incluso, explicar la relación entre actividad y pasividad asumida por el individuo en un acto amoroso (1920: 374-384). Otra hipótesis es la de la homosexualidad como huida del incesto (1920: 432). No obstante, Stekel considera éste un proceso en extremo complejo y de naturaleza más heterogénea que unilineal.

Schofield (1965) consideró a tales efectos del ambiente infantil (madre sobreprotectora y/o ausencia del padre) como contenedores de cierta validez en el análisis del origen de la homosexualidad. Sin embargo, aseguró que «si un niño se convierte en homosexual, siempre sería posible encontrar algo en las condiciones familiares de ese niño que ha sido propuesto, al menos por algunos escritores, como la causa de la homosexualidad» (1965: 261).

2. Si en determinado momento la preocupación estuvo dirigida a determinar cómo una educación familiar podría influir en el origen de la homosexuali-

3. Cfr. Robledo, 2000, epígrafe 1.1. (3). 
dad, hoy algunos estudios trazan su objetivo hacia el conocimiento de cómo la familia obstruye o facilita el proceso de adopción de una identidad gay o lesbiana. Newman y Muzzonigro (1993) han sido de los interesados en reconocer la influencia de los valores familiares (y la raza) en el proceso de coming $o u t^{4}$ principalmente en la adolescencia.

El coming out ha sido operacionalizado a partir de tres áreas: 1) sensibilización; 2) adopción de culpa y vergüenza, y 3) aceptación. Estos autores refieren algunas investigaciones en las cuales se ha demostrado la presencia del coming out en cualquier estadio del ciclo de vida del hombre (y la mujer), pero cuando ello sucede en las etapas más tempranas de la vida, todo comienza por la experimentación de un sentimiento de diferenciación, clasificado también como «sensibilización». Lo característico de esta etapa es que el niño no comprende el significado de tal sentimiento.

Durante la pubertad, se comienzan a hacer conscientes las primeras atracciones hacia personas de su mismo sexo. Estas experiencias pueden provocar sentimientos de culpa cuya defensa más común es la negación y represión del impulso homosexual. A esta etapa se le ha denominado período de «aislamiento social, emocional e informacional», de "confusión dolorosa» y de "culpa» o "vergüenza», cuya peculiaridad radica en una no comprensión sobre el significado de ser lesbiana o gay.

Tal «confusión» puede ser eventualmente resuelta. Esa resolución puede ir dirigida o a la inhibición de todo sentimiento homosexual o hacia una autoaceptación e integración a una identidad gay. Minton y McDonald — citados por Newman y Muzzonigro- afirman el paso o la transferencia interna de un role identity hacia un ego identity, esto es, se transita de una autoconsciencia de ser homosexual hacia un agradecimiento por serlo.

Newman y Muzzonigro explican que en la mayoría de las instituciones sociales se enseña que la homosexualidad es incompatible con los valores tradicionales, lo que provoca un fuerte conflicto en el adolescente y el joven en la adopción de una identidad homosexual. Este conflicto es mayor si los valores familiares crean expectativas en el joven en torno al matrimonio, la tenencia de hijos y los roles de géneros. Al ser la homosexualidad presentada como un detrimento de los valores tradicionales de la familia, el joven puede llegar a perder toda comunicación con los miembros de este grupo (1993: 216).

3. Dado su carácter no procreativo, también se ha asociado la homosexualidad con la absurda idea de una posible extinción de la especie humana en la Tierra. «Si todo el mundo se vuelve homosexual...»o «si pasa de fenómeno minoritario a norma grupal...» son frases que tratan de legitimar la necesidad de contener cualquier «liberación absoluta...» o "propagación...» de la homosexualidad fundada en la imposibilidad de procreación (biológica) de estas

4. Coming out es el término utilizado en inglés para referirse a la adopción de una identidad gay o lesbiana. En los países de habla hispana se suele utilizar el de «salir del armario». 
prácticas. Sin embargo, es totalmente improbable que en una sociedad «todo el mundo se vuelva homosexual...», como tampoco "todo el mundo es heterosexual ${ }^{5}$. Pero suponiendo que tal cosa suceda, igual como se ha distinguido entre acto y condición (o identidad) homosexual también pudiera distinguirse entre acto y condición heterosexual. En una sociedad donde «todo el mundo sea homosexual» existirían dispositivos que garantizarían actos heterosexuales con este fin: la procreación y conservación de la especie. Pero creemos que tal sociedad no existe y probablemente no haya existido ni existirá. El problema va más allá de la hipótesis de construcción de sociedades con una norma homosexual. Sencillamente, se sigue considerando el orden social prevaleciente como un orden natural y universal y se olvida que su evolución haya sido dada para legitimar formas de dominación y acumulación de poder.

4. Bajo el criterio anterior, a la familia, en su concepción tradicional, se le ha adjudicado, como función primera, la procreación y educación de la descendencia. Según Mohr (1993), la imposibilidad de tener hijos es muchas veces vista en el sentido de una incapacidad para obtener algo que es esencialmente parte de la riqueza de la vida. Antiguamente se sentía piedad hacia parejas con tal impedimento. Precisamente una relación homosexual tiene como impedimento la imposibilidad de procrear y al no poder cumplir la función «natural» de la unión sexual, queda fuera del orden familiar hegemónico.

Pero este orden jerárquico ha venido siendo cuestionado, no sólo en espacios académicos y políticos, sino también en las prácticas mismas de la vida sexual. La generación de descendencia ha cedido a la satisfacción sexual y ésta se ha constituido en el principal valor a considerar en la unión de parejas, junto, por supuesto, a los psicoafectivos.

Por otra parte, la familia se constituye formalmente a través de la institución del matrimonio. La posibilidad de unirse a través de este acto conlleva una serie de regulaciones, tales como las restricciones para que éste sea efectuado por parientes más o menos cercanos (incesto) o pertenecientes a un mismo tótem -en el caso de culturas con tal tradición-. Pero lo aparentemente menos natural y, por tanto, rara vez cuestionado, es la posibilidad de unión conyugal entre personas del mismo sexo.

En países donde se ha superado la disputa sobre la legitimidad de la pareja homosexual, la pregunta se ha dirigido hacia determinar si son o no son de igual naturaleza éstas en relación con las heterosexuales y si deben, por tanto, tener similar legislación o si necesitan de la creación de estructuras jurídicas particulares. Medina (1998) considera que la pareja homosexual es naturalmente distinta a la heterosexual y, por tanto, no resulta pertinente autorizar a los primeros a contraer matrimonio, al ser esta institución concebida para los segundos. Di Lernia e Iglesia (1998) coinciden con este criterio y postulan lo que dan en llamar «registro de convivencia». Ello consiste en «el asiento, median-

5. Véanse tablas Kinsay-Lizárraga en Robledo, 2000. 
te acta notarial [...] de la decisión de los interesados en formalizar su convivencia» (1998: 185).

Pero lo que no acepta ninguno de estos autores es la posibilidad de educación y cuidado de niños bajo el tutelaje de parejas homosexuales. Wagmaster y Beckerman (1998) enuncian una serie de criterios sobre los cuales se basa tal negativa. El primero de ellos es la supuesta predisposición a ser homosexual de un niño criado bajo estas condiciones, además de la proclividad a experimentar problemas emocionales, psicológicos y sociales asociados al comportamiento sexual de los padres. Se alude la imposibilidad de proporcionar estabilidad emocional al niño dada su alta promiscuidad y "actitud no saludable hacia el sexo opuesto"; el aumento de la probabilidad de riesgo de verse involucrado el niño en abuso sexual por parte de uno de sus tutores (padres), así como de caer en otras conductas antisociales supuestamente vinculadas a la homosexualidad, etc.

Sin embargo, los propios Wagmaster y Beckerman, en su ponencia, desmienten tales afirmaciones y señalan las ventajas que podrían tener niños y niñas criados dentro de parejas homosexuales. Entre ellos, el obtener un mayor aprecio por la diversidad humana; visión menos estrecha de la división del trabajo incluidos los roles de género; mayor sensación de afecto debido a las barreras a superar por gays y lesbianas, etc. ${ }^{6}$.

En Cuba, el debate no existe, aunque parece emerger como problema ${ }^{7}$. El Código Civil vigente en Cuba en 1938 (Menéndez, 1938: 69) recogía como uno de los impedimentos naturales del matrimonio la diferencia de sexo, lo cual se justificaba por el hecho natural de la impotencia que dos personas del mismo sexo tienen para realizar lo que era considerado su función principal: la procreación. Aunque el Código de Familia cubano, aprobado el 14 de febrero de 1975 , no mantuvo como impedimento la imposibilidad de generar descendencia por cualquier motivo ${ }^{8}$, en su artículo 2 primer párrafo establece que «el matrimonio es la unión voluntariamente concertada de un hombre y una mujer con aptitud legal para ello a fines de hacer vida en común» (Ley 1289/75: 7, la cursiva es nuestra).

Sin embargo, ¿existe una real demanda por parte de los grupos homosexuales cubanos de reconocimiento legal de la conformación de sus parejas?,

6. Estos autores concluyen: «[...] Dado que todos los seres tienen derecho a la libre expresión (primer Derecho) de la identidad de género que definan (segundo Derecho) para sí mismo, y el derecho a expresar libremente su identidad de género mediante prácticas sexuales concretadas entre dos adultos, todos los seres humanos tienen el derecho correspondiente a relaciones amorosas con compromisos mutuos con otros seres humanos y a celebrar contratos maritales, sin importar la forma cromosómica, los genitales, el sexo que se les asignó al nacer o el rol de género que desempeñaron en sus primeros años de vida cualquiera de los participantes en el vínculo» (1998: 165-166).

7. En un encuentro que sostuvimos con presidentes de tribunales civiles municipales de Ciudad de La Habana nos sorprendimos por el número de casos presentados de divorcio y custodia de hijos donde se hacía explícita la homosexualidad de uno o de ambos demandantes.

8. Véase la entrevista a la Dra. Olga Mesa en Robledo, 2000: anexo IV. 
¿es, en definitiva, pertinente hablar de "grupos homosexuales cubanos» como identidad particular, estructurada alrededor de elementos de cohesión grupal?

\section{El caso cubano}

\section{Proceso metodológico}

La unidad de observación la constituyeron jóvenes homosexuales varones de Ciudad de La Habana. Dado el carácter exploratorio de la investigación, la naturaleza del objeto y la unidad de observación, asumimos una metodología cualitativa con intención analítico-descriptiva y no experimental de correlación de variables o determinaciones causales entre éstas.

La exploración se concibió en tres momentos: primero, una observación de lo que definimos como «vida gay» en Ciudad de La Habana. Partimos de una observación inicial en la esquina de L y 23 , zona ampliamente conocida donde se concentra una buena parte de los jóvenes homosexuales, en la cual mantuvimos conversaciones informales con el objetivo de encontrar información que nos permitiera luego elaborar una guía de observación con un carácter más estructurado. Toda la información fue recogida en un registro de observación. Esta técnica nos permitió, además, elegir a un grupo de homosexuales para conocer más en profundidad sus criterios acerca de la relación entre homosexualidad y familia. Para ello, concebimos la realización de cuatro grupos de discusión, cantidad establecida bajo el criterio de disponibilidad de participantes y saturación de la información en el cuarto grupo. Como complemento, se realizaron dos entrevistas en profundidad a manera de historia personal a jóvenes con antecedentes de constitución de pareja (matrimonio) heterosexual.

\section{Homosexualidad masculina en La Habana}

En La Habana, al igual que en otros lugares de Cuba y del mundo, los homosexuales han desarrollado un lenguaje común, donde ciertas palabras y expresiones de carácter verbal o no verbal identifican caracteres físicos, conductuales o hechos de la vida cotidiana que pasan inadvertidos, carecen de sentido o son totalmente incomprensibles para aquéllos que no comparten sus simbologías. Ello se suma a una manera particular — por distinguirse del resto de la población - de compartir espacios públicos y otorgarles significados a cada uno de éstos sobre la base de las acciones y fines más comúnmente realizadas en los mismos. Los "puntos de encuentro» de los homosexuales varones en La Habana, en tanto que grupo, son primordialmente nocturnos: una esquina, una casa, un espacio del malecón, un parque, todos se convierten en espacios gay durante la noche. Algunos son comunes entre grupos de homosexuales y no homosexuales, donde ser identificado dentro de los primeros o los segundos depende más de los vínculos establecidos por el individuo con uno $\mathrm{u}$ otro grupo que por participar en el propio espacio; en otros, en cambio, el 
propio espacio es quien le otorga al individuo características y cualidades específicas a partir de los prejuicios ya construidos socialmente (también desde lo grupal) con relación a las personas que allí comparten. Ciertos espacios se configuran bajo la noción de estar; otros bajo las de pasar y buscar, o buscar y hacer. En algunos, los homosexuales no manifiestan su condición de manera abierta; en otros, se amplifican en su total expresión; pero todos sirven como guía sobre la cual se puede organizar parte de la vida cotidiana de un joven varón homosexual en La Habana. Espacios y palabras configuran la «vida gay» en la ciudad; no legitimados aún por la sociedad - aunque no totalmente desconocidos ni muchos menos indiferentes a los mecanismos de control social—, pero que existen para él y para sus iguales, pues él (ellos) los crean en sus prácticas cotidianas y van configurando y dibujando parte de la geometría social que viene forzando el cambio del sistema del cual forma parte. A estas estructuras está enlazado el gay en la Ciudad, como en un acoso, y donde se abre al reconocimiento de sí mismo y del grupo al cual se siente que pertenece?

\section{Familia y homosexualidad}

Los jóvenes homosexuales varones de Ciudad de La Habana objeto de este estudio expresan una noción de homosexualidad desde un discurso liberador, donde la pregunta del origen deja de ser problemático para pasar a reconocer su condición como aspecto natural de sus vidas. En ningún caso apareció la familia como origen de la homosexualidad y nadie expresó experiencias que la vinculen con su condición. El origen es descrito a partir de una determinante biológica, sea ésta hormonal, genética o de otro tipo, pero nunca asociado a patologías o deficiencias de cualquier índole. Éste, sin embargo, aparece al evaluar un "otros homosexuales» y ello pone en evidencia una clara distinción en la cual aparece, en un extremo, el «homosexual bueno» y, en el otro, el «homosexual malo». Los primeros se asocian con valores tales como vida saludable, integración y concordancia de género; los segundos, con vida no saludable, marginación y discordancia de género. Los propios jóvenes manifiestan una inconformidad por ser ésta última la imagen más fuertemente fijada en el imaginario social.

La noción sobre familia en los grupos estudiados se mueve en un eje cuyos extremos son lo biológico, aquello definido por lo «natural» y que establece los grados de parentesco por relaciones de consanguinidad, y lo relacional, cuya definición es más ambigua, al establecerse por prácticas individuales de coafectividad y con mayores dificultades de ser legitimado socialmente como tal. La noción de familia también se expresó en un eje valorativo cuyos puntos opuestos son el equilibrio y el conflicto. En el primer extremo, se condensan

9. Una descripción y análisis más amplios sobre estos espacios puede verse en Robledo, 2000, epig. 3.2. 
las asociaciones sobre la familia como espacio de seguridad, estabilidad y ayuda (material y espiritual); el segundo se construye a partir de la presencia de elementos distorsionadores sobre los cuales se configuran contradicciones y conflictos a niveles cotidianos entre sus miembros, o entre éstos y agentes externos. Todos estos significados se unen para considerar a la familia como importante y necesaria para la vida de cualquier ser humano, y entre éstos no está excluido el homosexual. La relación entre equilibrio y conflicto se expresa entre:

— «Familia biológica», con base en lazos de consanguinidad, y «familia relacional», con base en vínculos afectivos.

— «Familia real» (la que se tiene) y «familia ideal» (la que se quisiera tener).

— «Proyecto familiar»y "proyecto individual» (equilibrio o conflicto intergeneracional).

— «Proyecto de familia ideal» $\mathrm{y}$ «familia posible o realmente constituida».

Del análisis de los discursos, se desprende que las instituciones sociales refuerzan la noción de salud y enfermedad y el estereotipo del «homosexual malo» (marginal, afeminado y de prácticas no saludables). Ésta se fija en el espacio familiar junto a las tradicionales normas y expectativas con relación a las pautas de género (masculino) unidas a los mitos sociales sobre el significado de «ser homosexual». Por otra parte, la representación hegemónica de familia hace de ésta una estructura conservadora con tendencia al equilibrio y concebida, incluso por los propios jóvenes homosexuales objetos de nuestro estudio, como representante de lo tradicional, de lo estático, de lo biológico y jurídicamente establecido. La homosexualidad viene a presentarse entonces como elemento de disgregación de esa tradición o normalidad y se expresa como el rompimiento de una continuidad natural, y ello la hace inscribirse en el espacio de la transgresión. La relación entre homosexualidad y familia se refleja en un eje de equilibrio y conflicto, donde el primer extremo se va a manifestar en función de una continuidad del orden familiar tradicional y el segundo, como una transgresión o ruptura de dicho orden. La construcción del «sí mismo» homosexual tiene, así, un primer distanciamiento con el discurso familiar donde la noción de homosexualidad se mueve dentro de las categorías de salud y enfermedad, y moralidad y vicio. Al no tener otra referencia que no sea la de esta paridad dicotómica proveniente de los dispositivos legitimados socialmente como productores de verdad (entre ellos, la propia familia), el espacio de socialización del homosexual concluye junto a aquéllos con igual conflicto y se crea un grupo de referencia («amistades gay») que en un primer momento aparece como contraposición a la estructura familiar de origen. De esta manera, el joven comienza a moverse entre dos pares: adaptación e integración a la normalidad / inadaptación y exclusión de la normalidad. Apostar por uno o por otro siempre se hace teniendo en cuenta a la familia.

Cuando el «ser homosexual» entra en el marco de la familia, desde el descendiente, lo que marca toda la relación es un sentido de culpa y un sentido de 
vulnerabilidad. Ambos aparecen en dos direcciones: del homosexual a la familia y de la familia al homosexual. Del homosexual a la familia, el sentido de culpa se expresa en un reconocimiento de que su condición hace vulnerable a la familia por ser objeto de evaluación social. Ello se manifiesta en un propósito explícito de demostrar que no se pertenece al tipo de homosexual ("malo») fijado en el imaginario social sobre tales grupos. Todo lo anterior conduce a la adopción, por parte del joven homosexual, de un doble sentimiento de culpabilidad en la familia y la obligación de demostrar la capacidad de cumplir determinados roles y no perder así su estatus dentro de la estructura familiar.

De la familia al homosexual, se tiene culpa por no haber detectado el «problema" a tiempo y haberlo "corregido». Se culpa a algún miembro de la familia por no haber actuado adecuadamente en función de sus roles (madre que no supo educar o padre que no estuvo presente); se culpa a Dios y al destino. Tal sentimiento genera inmediatamente una búsqueda del origen. Se recogen del discurso social las nociones de salud y enfermedad, moralidad y vicio, y se expresan desde este punto las distintas respuestas posibles a la pregunta "iPor qué salió (es) así...?». La idea de patología (psíquica o somática) le imprime un sello de temporalidad y posible cura. De ahí el convencimiento de que, si se encuentra el origen, quizás se pueda «revertir» la «enfermedad». Tras el sentimiento de culpa, aparece el sentimiento de vulnerabilidad. La familia percibe que uno de sus miembros es vulnerable frente a los distintos espacios sociales. Pero quizás uno de los que más se manifiesta es el del temor al contagio de infecciones de transmisión sexual, reforzado con la noción que vincula directamente al sida con la homosexualidad.

\section{Proyecto}

Entre los jóvenes homosexuales varones participantes en el estudio, la posibilidad o no de conformar una pareja homosexual bajo el concepto de familia se inscribe en correspondencia con sus nociones sobre dicha estructura. Así, desde la comprensión biológica, donde la reproducción de la especie se convierte en el fin primero de la unión entre dos personas, tal pareja queda excluida; sin embargo, desde la noción que privilegia las relaciones de coafectividad, donde lo biológico no se excluye, pero no funge como condición necesaria, la relación entre personas del mismo sexo puede ser incluida dentro de una definición de esta institución social. En relación al eje entre equilibrio y conflicto, éste se constituye a partir de los distintos modelos posibles de llevar a vías de hecho un vínculo de pareja o construcción de familias dentro del marco de la homosexualidad.

La noción de conflicto tiene también su reflejo en los espacios familiar, social-institucional, social-comunitario y grupal. A éstos se les suman otras limitaciones propias de la juventud y algunas más particulares en relación al sector con preferencia homosexual. Las mismas se relacionan con los de orden material (vivienda) y espiritual corporal (angustias, celos, desencuentros), 
los prejuicios y mitos raciales y clasistas sobre la elección de pareja y la inexistencia de recursos legales que legitimen jurídicamente a la pareja homosexual.

Otra de las limitaciones aparecidas dentro de los grupos de discusión es la imposibilidad de tener descendencia junto a la pareja homosexual. Frente a las distintas variantes posibles para lograr un fin como éste, las posiciones son visiblemente contrapuestas. Cualquiera que sea el caso, vuelve a aparecer el sentido de vulnerabilidad, pero esta vez dirigido desde el homosexual hacia el hijo. Quienes apoyan la idea y expresan abiertamente el interés por un proyecto de este tipo, argumentan un sentimiento de paternidad innato y sin posibilidad alguna de ser sustituido por ningún otro vínculo afectivo. Se reconoce la vulnerabilidad, pero se considera que, con una educación adecuada, se podría palear cualquier situación de conflicto emocional del niño cuando se enfrente a evaluaciones y críticas provenientes de lo social, cualquiera sea su grado de cercanía con el menor. Quienes la rechazan, argumentan la necesaria responsabilidad asumida por cualquier individuo ante una decisión de este carácter. Ello implica necesariamente una represión de la tradicional «vida gay» que lleva el individuo (o la pareja), no solamente por un problema de presupuesto de tiempo, sino porque debe guardar una imagen frente a la sociedad y su hijo de tal manera que no afecte a éste último su normal desarrollo.

\section{Conclusiones}

El estudio evidenció la necesidad impostergable de desarrollar en Cuba una cultura de la diversidad donde se favorezcan los valores de inclusión y no de exclusión. Permitir, asimismo, en espacios públicos de recreación y consumo, la permanencia de parejas y su libre expresión como tales, cualquiera sea la preferencia sexual de sus miembros y donde lo punible no se vincule a esta última condición. Para ello, es imprescindible incluir en la legislación cubana la no discriminación por preferencias sexuales; desarrollar programas de educación sexual y de pareja, dirigidos a grupos de jóvenes homosexuales, o programas generales hacia la juventud, donde se incluyan las especificidades correspondientes a este tipo de relación; favorecer en ellos las nociones de afectividad y responsabilidad sobre las cuales debe basarse cualquier construcción familiar, incluida la educación y el cuidado de la descendencia, y, por último, poner bajo estudio y crear recursos legales sobre los cuales pueda legitimarse jurídicamente, en Cuba, una pareja homosexual.

\section{Bibliografía}

ADLER, Alfred (1930). El problema del homosexualismo y otros estudios sexuales. 2a ed. Barcelona: Apolo, 1936 ( $2^{a}$ ed.).

BlanCO, Juan A. (1995). Tercer Milenio. Una visión alternativa a la postmodernidad. La Habana: Fundación Félix Varela. 
Di LeRnia, María C.; Iglesias, María G. (1998). «Conviviencia homosexual ¿̨una nueva forma familiar?». En X Congreso Internacional de Derecho de Familia «El Derecho de Familia y los nuevos paradigmas". Comisión no 4 "Diversas formas familiares». Mendoza, Argentina, 20 al 24 de septiembre, p. 181-187.

Ley $n^{\circ}$ 1289/75, Código de Familia. Ciudad de La Habana: Imprenta Central de las FAR, 1987.

Medina, Graciela (1998). «El Derecho de Familia y los nuevos paradigmas. Diversas formas familiares. Uniones de hecho homo y heterosexuales». En $X$ Congreso Internacional de Derecho de Familia "El Derecho de Familia y los nuevos paradigmas». Comisión no 4 "Diversas formas familiares», Mendoza, Argentina, 20 al 24 de septiembre, p. 322-331.

MENÉNDEZ, Emilio (1938). El matrimonio. La Habana, s/e.

MoHR, Richard (1986). "Gay Basics: Some Questions, Facts and Values». En SHAw, William, H. (ed.). Social and Personal Ethics. Belmont, California: Wadsworth Publishing Company, 1993, p. 358-368.

Newman, Bernie; Muzzonigro, Peter G. (1993). «The effects of traditional family values on the comming out process of gay male adolescents». Adolescense, vol. 28, no 109 , primavera, p. 213-226.

Robledo, Luis (2000). Homosexualidad y familia: acoso y simetrías. Universitat Autònoma de Barcelona. Universidad de La Habana. Ciudad de La Habana (tesis de maestría).

SCHOFIELD, Michael G. (1965). Aspectos sociológicos de la homosexualidad; un estudio comparativo de tres tipos de homosexuales. Barcelona: Fontanella, 1969.

STEKEL, Wilhelm (1920). Onanismo y homosexualidad: la neurosis homosexual. Buenos Aires: Ediciones Imán, 1952.

STEKEL, Wilhelm (1924). Sadismo y masoquismo. Buenos Aires: Ediciones Imán, 1954.

WaGMASTER, Adriana; BeCKERMAN, Jorge M. (1998). «Niños criados por parejas homosexuales: caracterización sociojurídica de su contexto. Hacia una construcción de la relación coparento filial para que el derecho adopte a esos niños». En $X$ Congreso Internacional de Derecho de Familia "El Derecho de Familia y los nuevos paradigmas». Comisión no 4 "Diversas formas familiares». Mendoza, Argentina, 20 al 24 de septiembre, p. 138-180. 